bioRxiv preprint doi: https://doi org/10.1101/2022 02.22481461 ; this version posted February 22,2022 . The copyright holder for this

\title{
Characterization of Arabian Peninsula whole exomes: exploring high inbreeding features
}

Joana C. Ferreira ${ }^{1,2,3}$, Farida Alshamali ${ }^{4}$, Luisa Pereira ${ }^{1,2}$, Veronica Fernandes ${ }^{1,2}$

1 i3S - Instituto de Investigação e Inovação em Saúde, Universidade do Porto, Porto, Portugal

2 IPATIMUP - Instituto de Patologia e Imunologia Molecular da Universidade do Porto, Porto,

Portugal

3 ICBAS - Instituto de Ciências Biomédicas Abel Salazar, Universidade do Porto, Porto, Portugal

4 Department of Forensic Sciences and Criminology, Dubai Police General Headquarters, Dubai, United Arab Emirates

Keywords: Arabian Peninsula; whole exome sequences enriched with UTRs; new variants; pathogenic inference; consanguinity 


\begin{abstract}
The exome (WES) capture enriched for UTRs on 90 Arabian Peninsula (AP) populations contributed nearly 20,000 new variants from a total over 145,000 total variants. Almost half of these variants were in UTR3, reflecting the low effort we have dedicated to cataloguing these regions, which can bear an important proportion of functional variants, as being discovered in genome-wide association studies. By applying several pathogenic predicting tools, we have demonstrated the high burden in potentially deleterious variants (especially in nonsynonymous and UTR variants located in genes that have been associated mainly with neurologic disease and congenital malformations) contained in AP WES, and the burden was as high as the consanguinity level (inferred as sum of runs of homozygosity, SROH) increased. Arabians had twice SROH values in relation to Europeans and East Asians, and within AP, Saudi Arabia had the highest values and Oman the lowest. We must pursuit cataloguing diversity in populations with high consanguinity, as the potentially pathogenic variants are not eliminated by genetic drift as much as in less consanguineous populations.
\end{abstract}




\section{Introduction}

The technological developments introduced with the next-generation sequencing methodology are allowing the high throughput characterization of millions of polymorphisms, enhancing the knowledge on genetic diversity between and within populations, and enabling new lens to explore the genetic basis of diseases [1,2]. Directed sequencing of all exons (known as whole exome sequencing, WES) is being increasingly used in clinical genetics [3], as these regions are probable locations of candidate alleles conferring susceptibility to monogenic and complex diseases. International consortia have been collecting big exome data and making them publicly available for mining of variants [4,5], such as the Exome Sequencing Project (ESP) Exome Variant Server (EVS) [6] representing more than 200,000 individuals from multiple ESP cohorts, and the Exome Aggregation Consortium (ExAC) database [7] spanning 60,706 unrelated individuals sequenced as part of various disease-specific and population genetic studies. The ExAC creators launched afterwards the Genome Aggregation Database (gnomAD), which englobes currently 125,748 WES and 15,708 WGS [8]. This big data is allowing to predict the functional effects of variants on diverse traits in different populations, and guiding inference of disease risk at an individual basis. However, WES catalogues are still extremely biased in terms of ancestry, being largely based on data from European and East Asian populations [4,9], which limits the transferability of findings to other population groups [10].

A region particularly understudied in large-scale sequencing projects is the Middle East and more specifically the Arabian Peninsula (AP). AP populations are amongst the most nonAfrican ancestral populations in the globe, as this region was the first outpost of the successful out-of-Africa (OOA) migration, at around 60 thousand years ago (ka) $[11,12]$. Concordantly, signatures of ancient ancestry were detected in extant AP populations, especially in the AraboPersian Gulf region: (1) as relic maternal lineages from the west-Eurasian haplogroups N1, N2 and X, branching directly from the root of macro-haplogroup N (Fernandes et al. 2012); (2) in the autosomal genome, the component known as basal Eurasian, identifiable when ancient DNA information is integrated in the analysis (Ferreira et al. 2021). There were clear continuums throughout time linking western Arabia with the Levant and Africa, and eastern Arabia with Iran and the Caucasus, making AP an important migration nexus between the main human population groups $[13,14]$. This high admixture enriched the Arabian genomes with non-autochthonous selected variants, mainly for malaria protection (higher in the west), lactose 
tolerance (European/South Asian-derived allele in the eastern AP) and other immune system defences (throughout the Peninsula) $[13,15]$.

Despite the high ancestry admixture in AP, Arabs have traditionally a high level of consanguinity, reaching an overall prevalence of 56\% in Saudi Arabia, from which $33.6 \%$ are first-cousin consanguineous marriages [16]. In addition to a high consanguinity rate, Arabs are also characterized by large family sizes and advanced paternal and/or maternal age [17], increasing the risk of congenital anomalies in their offspring [16]. These factors are associated with a high number of congenital and genetic disorders [17], such as impaired hearing (3.5 times higher in consanguineous than in non-consanguineous mating) [16] and Down's syndrome (Arab countries exceeds the 1.2-1.7 per 1000 typical for industrialised countries; $[17,18])$. Other complex disorders with a genetic component are also common throughout the Arab world, including haemoglobinopathies, glucose-6-phosphate dehydrogenase deficiency and metabolic diseases (obesity, type 2 diabetes and dyslipidemia), and all have been associated with the high level of consanguinity in this region [16]. The reason for this is that high level of consanguinity increases frequencies of rare variants and extends stretches of homozygous chromosomal fragments (long runs of homozygosity across all sites, ROH) [19]. Thus, the study of $\mathrm{ROH}$ length and burden of deleterious variation can provide important insights into the human demographic history and clinical applications [20,21]. Most of the WES available for Arabians were obtained in cohorts of patients [22-25]. Fewer WES are available for the general population: one obtained in Qatari populations identified eight hematologic variants, five metabolic, four eye-related, three inflammatory, three cardiovascular and three neurologic as the most common disease-related variants in those consanguineous cohorts [26]; an important cohort, the Greater Middle East (GME) Variome Project containing 2,497 individuals from 19 Arab and non-Arab Muslim countries [27] detected large and rare homozygous blocks, compatible with recent consanguineous matings, rendering easy to identify genes harbouring putatively high-impact homozygous variants.

As the effective size of the WES catalogue for AP general population remains low, in this work we performed a characterization of Arabian WES by randomly selecting 25 individuals from each AP country (Saudi Arabia, Yemen, United Arab Emirates and Oman). We enriched our WES with the sequencing of the untranslated regions 5 and 3 (UTR5 and UTR3), usually not included in these panels, as these regions play a role in the modulation of mRNA transcription, secondary structure, stability, localization, translation, and access to regulators like microRNAs and RNA-binding proteins [28]. We aimed to identify new and rare variants 
bioRxiv preprint doi: https://doi.org/10.1101/2022.02.22.481461; this version posted February 22, 2022. The copyright holder for this preprint (which was not certified by peer review) is the author/funder, who has granted bioRxiv a license to display the preprint in perpetuity. It is made available under aCC-BY-NC-ND 4.0 International license.

(especially in UTRs), to infer their possible functional impact, and to relate them with the consanguinity levels. 


\section{Materials and methods}

\section{Sample collection and WES capture}

We conducted WES of 94 individuals from AP (23 from Saudi Arabia, 24 from Yemen, 25 from Oman and 22 from UAE). These samples were part of a larger cohort of 420 Dubai residents who were born across the AP. As this cohort was previously analysed with the Illumina Human Omni Express Bead Chip containing 741,000 SNPs [13,29], it allowed us to selected the 94 individuals for WES based on the genomic information that they were nonrelated and non-recent migrants from sub-Saharan Africa. This study obtained the ethical approval from the Ethics Committee of the University of Porto, Portugal (17/CEUP/2012).

WES was performed at two companies: Macrogen Inc. (Seoul, South Korea) for 50 samples with a 100x average depth coverage; and STAB VIDA (Caparica, Portugal) for 44 samples with a 30x average depth coverage. The WES were captured using the SureSelect Human All Exon V5 + UTRs (50 Mb) target enrichment kit. Sequencing was performed with $2 \times 100 \mathrm{bp}$ paired end reads on Illumina HiSeq platform (Illumina, San Diego, CA, USA) according to manufacturer's protocol.

In order to have comparable values for other worldwide populations, the 1000 Genomes [30] WGS from African, European and East Asian populations (90 individuals randomly selected from each of these regions; Supplementary Table 1) were extracted, and variants located in the genomic regions covered by our WES (including regulatory untranslated regions UTRs) were considered for further analyses in this manuscript.

\section{Variant calling, filtering and annotation}

Paired-end reads were trimmed using the trimmomatic tool and aligned to human reference genome NCBI Build 37 using the Burrows-Wheeler Aligner (BWA v0.7.16a) algorithm. The paired-read alignments were sorted and stored in BAM format using samtools (v1.5). All the samples passed the quality control performed with FastQC and Alfred tools. Duplicates were marked and eliminated with Picard (v1.139), and local re-alignment and Base Quality Score Recalibration were carried out using the Genome Analysis Toolkit (GATK, v4.2.0.0, https://software.broadinstitute.org/gatk/). 
Variants were called using the Genome Analysis Toolkit (GATK v4.2.0.0) Haplotypecaller. A minimum value of $10 \%$ was set for missing genotype, leading to exclusion of three samples from further analyses. Variants were initially filtered to have a minimum depth of 7, Phred quality score $>30$, and genotype quality score $>20$, using the bcftools (v2.26.0). In the case of heterozygous callings for which the ratio of the less covered allele (reference or derived) over the total calls was $<25 \%$, the genotyping was corrected to homozygous of the most frequent allele, by using an in-house script. Finally, we excluded from the analysis multi-allelic variants, indels and positions for which more than $5 \%$ of the genotypes were missing. A principal component analysis (PCA) was performed using the SmartPCA tool from the EIGENSTRAT software package (v6.1.3), to identify potential batch effects between laboratories, or outliers. After the PCA, one sample was eliminated as it was a clear outlier. The final dataset contained 90 samples (23 from Saudi Arabia, 24 from Yemen, 24 from Oman and 19 from UAE). To further assess the quality of the calling, we evaluated in the bcftools stats the aggregate transition-to-transversion (Ti/Tv) ratio for all variants and exonic variants.

The ANNOVAR tool (version available in 2019-10-24; [31]) was used for the functional annotation of the called variants, and to verify if they were previously described in the following publicly available databases (downloaded on $10^{\text {th }}$ September 2021): WGS group, which included the GnomAD_WGS V2.0.1 (11st March 2017), dbSNP_142 (28 ${ }^{\text {th }}$ December 2014), Haplotype Reference Consortium (HRC; $3^{\text {rd }}$ December 2015), and 1000 Genomes $\left(24^{\text {th }}\right.$ August 2015); WES group, consisting in GnomAD_exome V2.0.1 (11st March 2017), NHLBIESP (esp6500siv2_all; 22 ${ }^{\text {nd }}$ December 2014) and ExAC (29 ${ }^{\text {th }}$ November 2015); ClinVar (clinvar_20160302; $3^{\text {rd }}$ October 2017) and dbNSFP3.0a group; and finally, Greater Middle East group (GME; $24^{\text {th }}$ October 2016).

\section{Functional constraint analysis}

The online CADD tool (v1.6; https://cadd.gs.washington.edu/snv) was used to evaluate the evolutionary conservation of AP (and comparable 1000 Genomes datasets) variants, through several metrics. The first type of metrics considered only alignment-based conservation values: (1) GERP or Genomic Evolutionary Rate Profiling [32], which evaluates non-neutral rates of substitution from multiple mammalian species alignments, and categorizes mutations by their predicted deleterious effect in neutral $(-2<$ GERP $<2)$, slightly deleterious $(2<$ GERP $<4)$, moderate $(4<\mathrm{GERP}<6)$, and extremely deleterious (GERP> 6) groups (categories according 
to [33,34]); (2) Phylop [35], also based on the alignment of mammalian species, for which a negative value indicates faster-than expected evolution, while positive values imply conservation.

Secondly, the metrics SIFT (Sorting Intolerant From Tolerant; [36]) and PolyPhen [37] that predict whether nonsynonymous substitutions are likely to have a deleterious effect on the protein function were investigated. A nonsynonymous variant with a SIFT score $<0.05$ will be classified as 'deleterious' while others are called 'tolerated' (benign) [38]. In contrast, PolyPhen 2 calculates the probability that a given variant will be 'benign' for scores less than or equal to 0.446 , 'possibly damaging' for scores greater than 0.446 and less than or equal to 0.908 , and 'probably damaging' for scores greater than 0.908 .

Finally, the integrative "scaled C-score" was considered. This score provides a ranking of variants more likely to be deleterious by integrating multiple annotations including conservation and functional information into one metric [39]. We applied a cutoff of 20, below which the variants were classified as benign and otherwise harmful, as suggested by the authors [39].

\section{Inbreeding features}

To infer the degree of relatedness between the 90 AP WES we used the KING tool [40] by estimating kinship coefficients and inferring IBD segments for all pairwise relationships. Unrelated pairs can be precisely separated from close relatives with accuracy up 4th-degree.

To assess the individual runs of homozygosity (ROHs), we estimated ROHs using the PLINK tool (version 1.9) following these published [41] parameters: a size threshold (kb) to call an ROH (homozyg-kb) of $1000 \mathrm{~kb}$; a SNP number threshold to call an ROH (Homozyg-snp) of 10 SNPs; a sliding window size in SNPs (Homozyg-window-snp) of 20 SNPs; allowing 5 missing SNPs (Homozyg-window-missing); with a proportion of homozygous windows threshold (Homozyg-window-threshold) of 0.05; a minimum SNP density of $200 \mathrm{~kb}$ to call an ROH (Homozyg-density); allowing a maximum gap (Homozyg-gap) of $4000 \mathrm{~kb}$; and allowing only 1 heterozygous SNP (Homozyg-window-het). For each AP individual included in the analysis, the sum of the length of ROHs ( $\mathrm{SROH}$ ) was calculated. These AP SROHs were used for testing the linear regression with the burden of predicted pathogenic non-synonymous variants (inferred for both SIFT and PolyPhen algorithms), and a f-statistic test was applied. 
Mining of diseases associated with highlighted genes

Information for disease associated with genes for which AP individuals presented predicted pathogenic non-synonymous and conserved UTR variants was collected from the OMIM (https://www.omim.org/) database. Then, these diseases were mined in the MalaCards: The human disease database (https://www.malacards.org/) for classification in broad categories. In cases of disease affecting several organs, decisions were made for inclusion in one category instead of another (Supplementary Table 2).

\section{Graphs and statistical tests}

The plots were built with the venn [42,43] and ggplot2 package [44] in R [45]. The calculations for those plots were performed through in-house R-scripts. 


\section{Results}

Diversity of the AP WES (enriched for UTRS)

The 90 AP WES presented 145,630 variants, around half the level of diversity displayed by the European (270,913 variants) and East Asian (254,527 variants) regions, and one third by subSaharan Africans (443,767 variants) (1000 Genomes populations, for an equal number of individuals and matching genomic segments). The transition-to-transversion ratio (Ti/Tv) was 2.59 for the entire dataset, and 3.23 when considering only the exonic variants as this latter value is more comparable with other high quality WES datasets not enriched for UTRs [1,2].

Of the total 145,630 variants, 19,701 (13.5\%) were new when compared against several public databases (Figure 1A). Among the known variants (Figure 1B; Supplementary Table 3), identical proportions (around a quarter) were shared between nonsynonymous, intronic and UTR3 classes, followed by synonymous $(\sim 16 \%)$ class, and rare proportions of all remaining classes (nonsense, UTR5, ncRNA, upstream/downstream, intergenic and splicing). Interestingly, for the new variants (Figure 1C), almost half of these were located in UTR3, followed still by $29 \%$ of intronic, $14 \%$ of synonymous and rare instances of the other classes of new variants. It is not surprising the high proportion of new variants in UTR3, and that the value of new variants in UTR5 is also twice the value for this class in known variants, as UTRs are rarely covered in WES screenings. We detected very few new nonsynonymous variants (57 $-<1 \%$ of total new variants), showing that saturation for this class of variants is almost reached for populations of mainly Eurasian ancestry.

Interestingly, a substantial amount of AP variants (7092) was only shared with clinically/functionally focused databases, namely ClinVar [46] and dbNSFP3.0a [47]. A careful inspection of these variants revealed they were mostly included in the dbNSFP3.0a dataset (88.8\% non-synonymous, $6.78 \%$ non-sense and $2.19 \%$ splicing variants), which prioritizes these type of variants from UK10K and ExAC datasets. These variants are of possible functional relevance, as we will see in detail in the next section. 
A

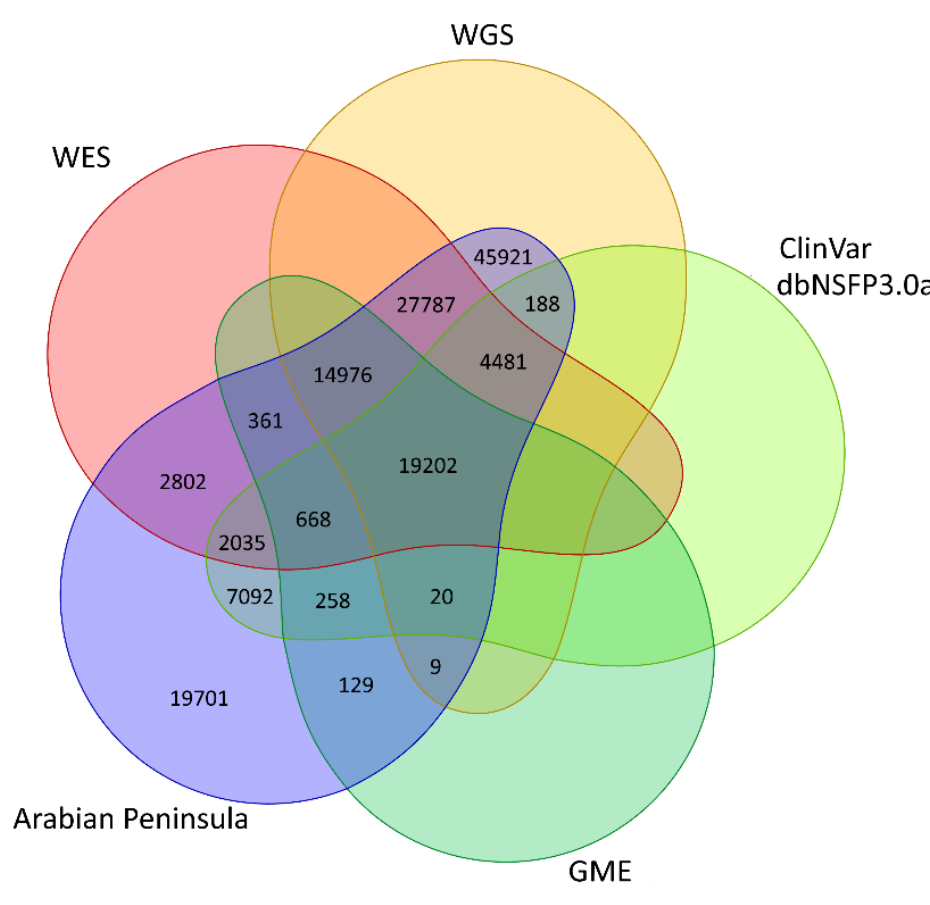

B Known Variants

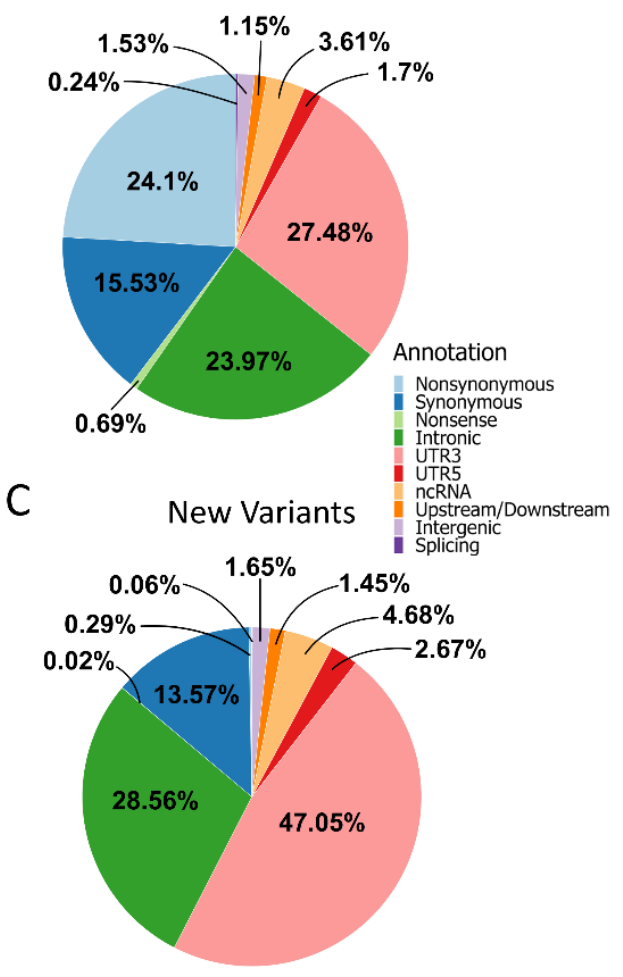

Figure 1: The global diversity observed in the 90 AP WES. A. Venn diagram illustrating new and shared variants between AP WES and publicly available databases (as referred in the material and methods section). B. Classes of known variants observed in the AP WES. C. Classes of new variants observed in the AP WES.

\section{Functional constraint features of AP WES}

The conservation score GERP (Figure 2A and B) allowed to confirm that AP had 83.9\% of variants classified either as neutral $(66.2 \%$ for GERP $<2)$ or as slightly deleterious $(17.7 \%$ for $2>$ GERP $>4$ ). These variants were broadly distributed by class of variants, testifying that most of the genome of AP is functionally neutral as expected. Comparatively, in the two most deleterious categories ( $4>$ GERP> 6 and $4>$ GERP> 6 ) there was an increase of nonsense, nonsynonymous and splicing variants, and a decrease of UTR5 and UTR3. The results from the Phylop algorithm, another conservation score, were consistent with GERP (Supplementary Figure 1). When comparing with the other worldwide regions (Figure 2C), AP displayed the higher amount of moderate and extremely deleterious variants $(15.31 \%$ and $0.81 \%$ respectively; compensated by the lower amount of neutral variants), followed ex aequo by 
bioRxiv preprint doi: https://doi.org/10.1101/2022.02.22.481461; this version posted February 22, 2022. The copyright holder for this preprint (which was not certified by peer review) is the author/funder, who has granted bioRxiv a license to display the preprint in perpetuity. It is made available under aCC-BY-NC-ND 4.0 International license.

Europe (11.22\% and $0.37 \%$ respectively) and East Asia (11.49\% and $0.39 \%$ respectively), and then Africa (10.18\% and $0.30 \%$ respectively) with the lower values.

\section{A}
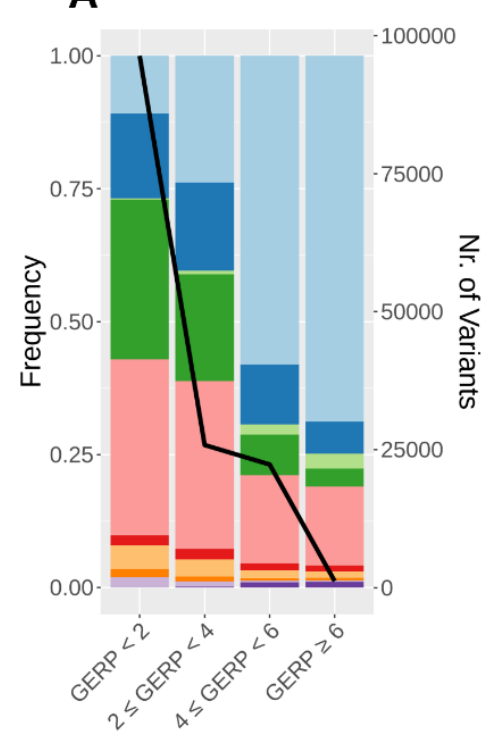

Nonsynonymous Synonymous

Nonsense Intronic
B

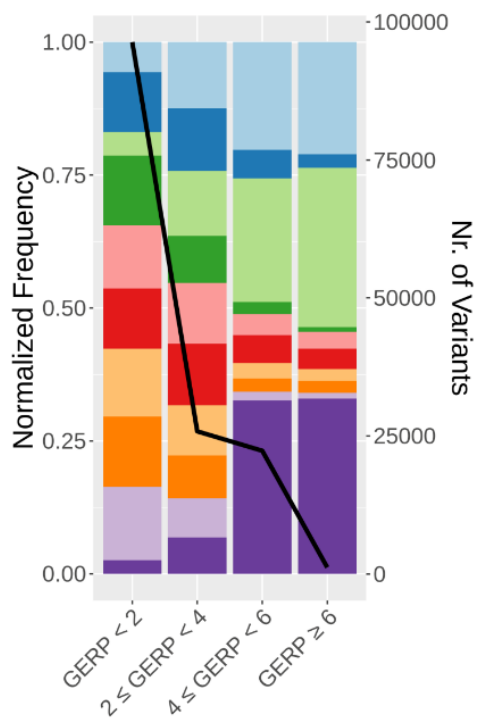

UTR3 ncRNA
UTR5 Upstream/Downstream

$\begin{array}{ll}\text { UTR3 ncRNA } & \text { Intergenic } \\ \text { UTR5 Upstream/Downstream } & \text { Splicing }\end{array}$

Splicing

D

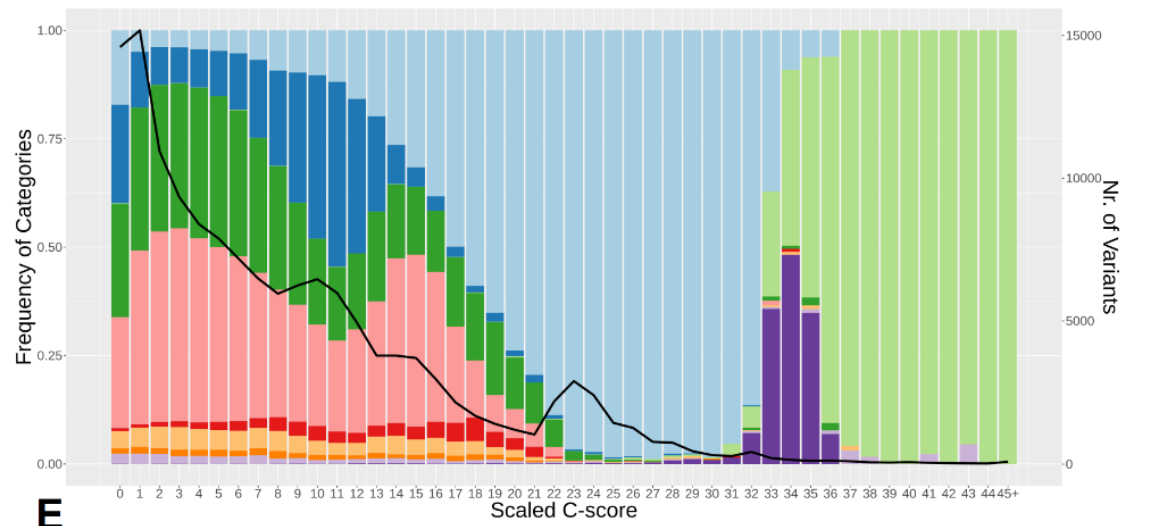

E

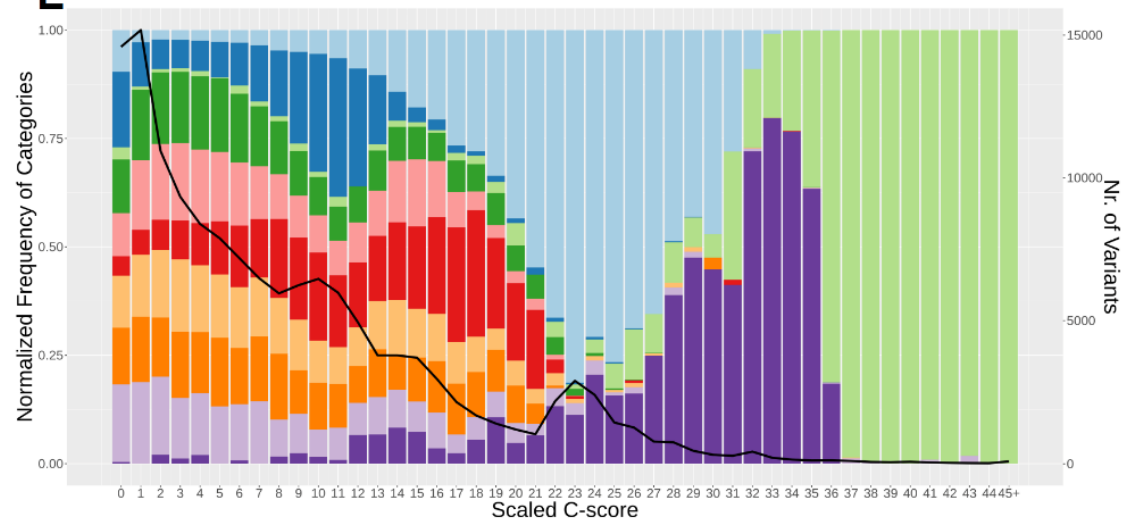

C

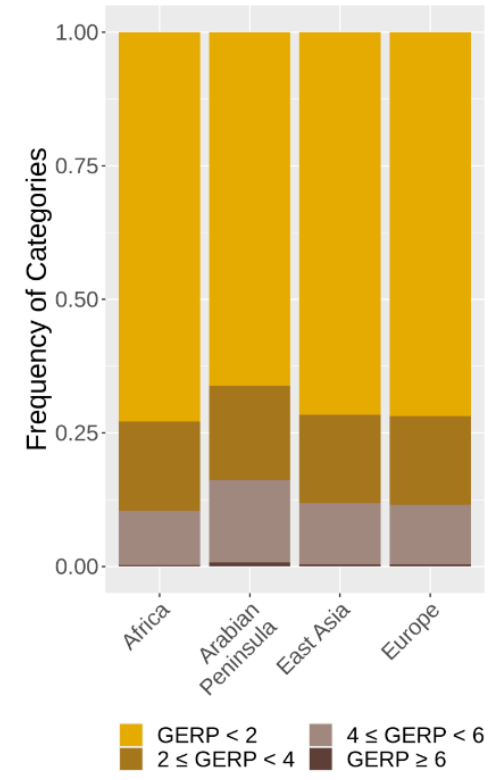

$\mathbf{F}$

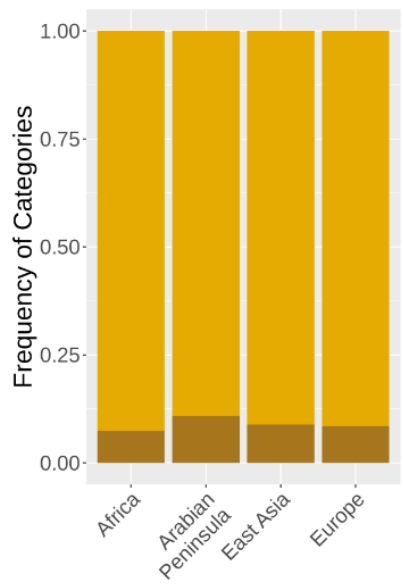

Scaled C-score $\square<20$ Categories: $\quad \square \geq 20$

Figure 2: Results for the conservation GERP and the integrative scaled C scores. A. Proportion of AP variants from each class of variants in each GERP category. The line represents the total 
number of variants in each score category (same meaning for B, D and E). B. Proportion of AP variants after normalizing by the total number of variants in each class, observed in that GERP category. C. Proportion of variants from each GERP category in AP and other worldwide regions. GERP values were classified into four groups: neutral $(2<$ GERP), slightly deleterious ( $2<=$ GERP $<4)$, moderate $(4<=$ GERP $<6)$, and extremely deleterious (GERP> 6). D. Proportion of AP variants from each class of variants along the scaled C-scores. E. Proportion of AP variants after normalizing by the total number of variants in each class, along the scaled C-scores. F. Proportion of variants with scaled C-scores below and above 20 (benign and harmful variants, respectively) in AP and other worldwide regions.

A similar pattern was observed for the integrative scaled C-scores, but now with better resolution (Figure 2D and E). Nonsense variants are the most extreme in the harmful scale, preceded by the splicing variants. For non-synonymous variants, a high percentage of them $(45,89 \%)$ displayed values equal or higher to 20 , so more prone to have functional impact. In the other extreme, variants that are of lower functional impact, were the synonymous, intronic, UTRs, and the rarer ncRNA, upstream/downstream and intergenic variants. Comparing with the other geographical regions (Figure 2F), again AP had a higher proportion of potentially harmful variants when inferred through the scaled C-scores $(10.86 \%)$ than the rest of the globe (East Asia-8.89\%; Europe - 8.52\%; Africa-7.38\%).

Focusing on the predicted pathogenicity for AP nonsynonymous variants, provided by SIFT and PolyPhen metrics (Figure 3A), a proportion of $15.56 \%(4,673$ out of 30,033 nonsynonymous variants for which values for both metrics were available) were inferred as pathogenic by SIFT and PolyPhen ("deleterious" and "probably damaging", respectively). These variants were distributed in 3,285 genes, and 756 of these genes have been associated with diseases of various types (Figure 3B), namely (in decreasing importance) neurologic, congenital malformation, metabolic, ear and eye, immune and infection-related, oncologic, cardiovascular and blood. Only six of these inferred as pathogenic nonsynonymous variants were new, and they were located in the genes: CYFIP1, associated with congenital malformation and neurologic diseases; ITGA10 and PIAS3, associated with oncologic, and ear and eye diseases; GNG14 involved in signalling pathways; C1GALT1C1L that enables the activity of a galactosyltransferase whose deficit is associated with congenital malformation; and FAM240A that is mainly expressed in brain. 

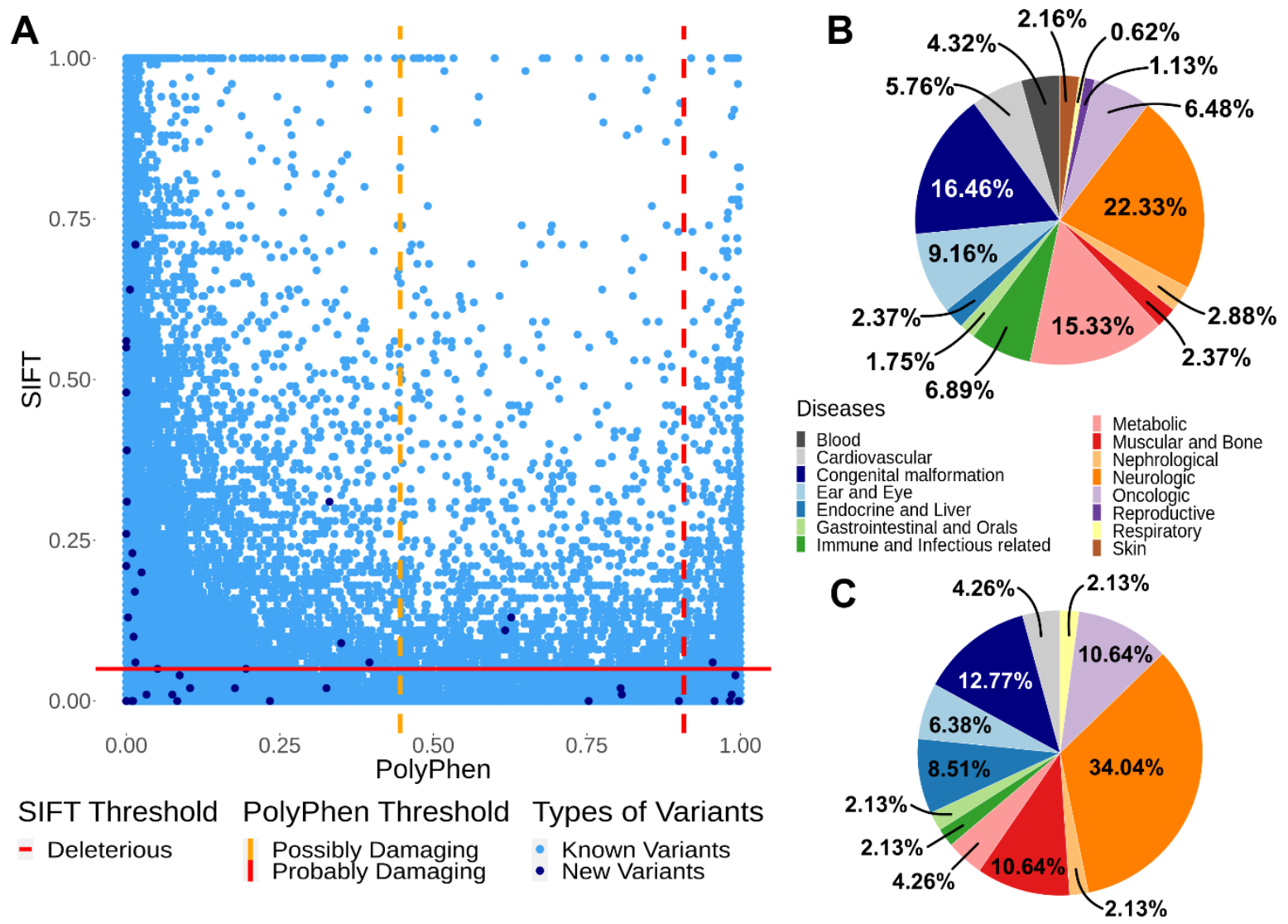

Figure 3: Pathogenic predictions. A. SIFT scores versus PolyPhen2 scores. The known variants are represented in light blue dots while new variants are represented as dark blue dots. The yellow dashed line correspond to the thresholds for predicting $0.446<$ Possibly Damaging $<0.908$ for PolyPhen2. The red dashed lines correspond to the thresholds for predicting deleterious variants: $<0.05$ for SIFT and Probably Damaging >0.908 for PolyPhen2.

B. Diseases associated with 756 of the genes bearing predicted pathogenic (by both SIFT and PolyPhen) non-synonymous variants in AP, according to OMIM (classification of disease based on MalaCards database; Supplementary Table 4). C. Diseases associated with 31 of the genes bearing non-conserved (C-score above 20) UTR3 and UTR5 variants in AP, according to OMIM (classification of disease based on MalaCards database; Supplementary Table 4).

Our AP WES had the special feature of being enriched for the UTR regions, providing additional information for these parts of the genome that, although untranslated, can be pivotal in the regulation of gene expression. As we have referred before, most of UTR variants are non-conserved, but focusing on the proportion of UTRs with C-scores above 20, $1.76 \%$ of UTR5 and $0.31 \%$ of UTR3 variants (47 out of 2671 UTR5 and 138 out of 43,868 UTR3) can be potentially harmful variants. These variants were distributed in 116 genes, and 31 of these 
genes have been associated with various types of diseases (Figure 3C), with the high percentage of them classified as neurologic diseases (34.04\%) and congenital malformation $(12.77 \%)$, compatible with the results observed for the inferred pathogenic nonsynonymous variants.

\section{Inbreeding features inferred from the AP WES}

The inference of relatedness between the 90 AP WES, based on the genomic kinship coefficient, revealed that all the individuals were classified as unrelated (up 4th-degree), as expected for a cohort representative of the general population. We then estimated ROHs longer than $1 \mathrm{Mb}$ in $\mathrm{AP}$ and other worldwide populations, in order to compare inbreeding features. In concordance with the known higher consanguinity of AP populations, there were more ROHs of all sizes in Arabia than in the other regions of the globe (Figure 4A), mainly for the ROH size category $2-4 \mathrm{Mb}$. The largest ROHs detected in each AP population were: Oman-70.4Mb; Yemen-59.8Mb; Saudi Arabia-41.2Mb; and UAE-26Mb.

Not only AP individuals had larger ROHs, but also the total sum of ROHs (SROHs) per AP genome was in mean twice the values observed in the individuals from other worldwide populations (Figure 4B): $1200 \mathrm{Mb}$ in AP; $720 \mathrm{Mb}$ in East Asians, $700 \mathrm{Mb}$ in Europeans; and $650 \mathrm{Mb}$ in Africans. Within AP, the highest values were observed in Saudi Arabia and the lowest in Oman. In terms of SROH distribution within each population group, there was a high heterogeneity between values from AP individuals (Supplementary Figure 2), while individuals from other regions were more homogeneous in this metric. This highest SRHOs values in each AP country were: Saudi Arabia-1969Mb; Yemen-1781Mb; UAE-1171Mb; and Oman-1152MB.

Interestingly, within AP there is a very significant positive linear regression $\left(\mathrm{r}^{2}=0.9712\right.$; $\mathrm{p}$ value $=1.315 \mathrm{e}^{-69}$ ) between SROHs and the burden of predicted pathogenic non-synonymous variants (inferred for both SIFT and PolyPhen algorithms; Figure 4C). 

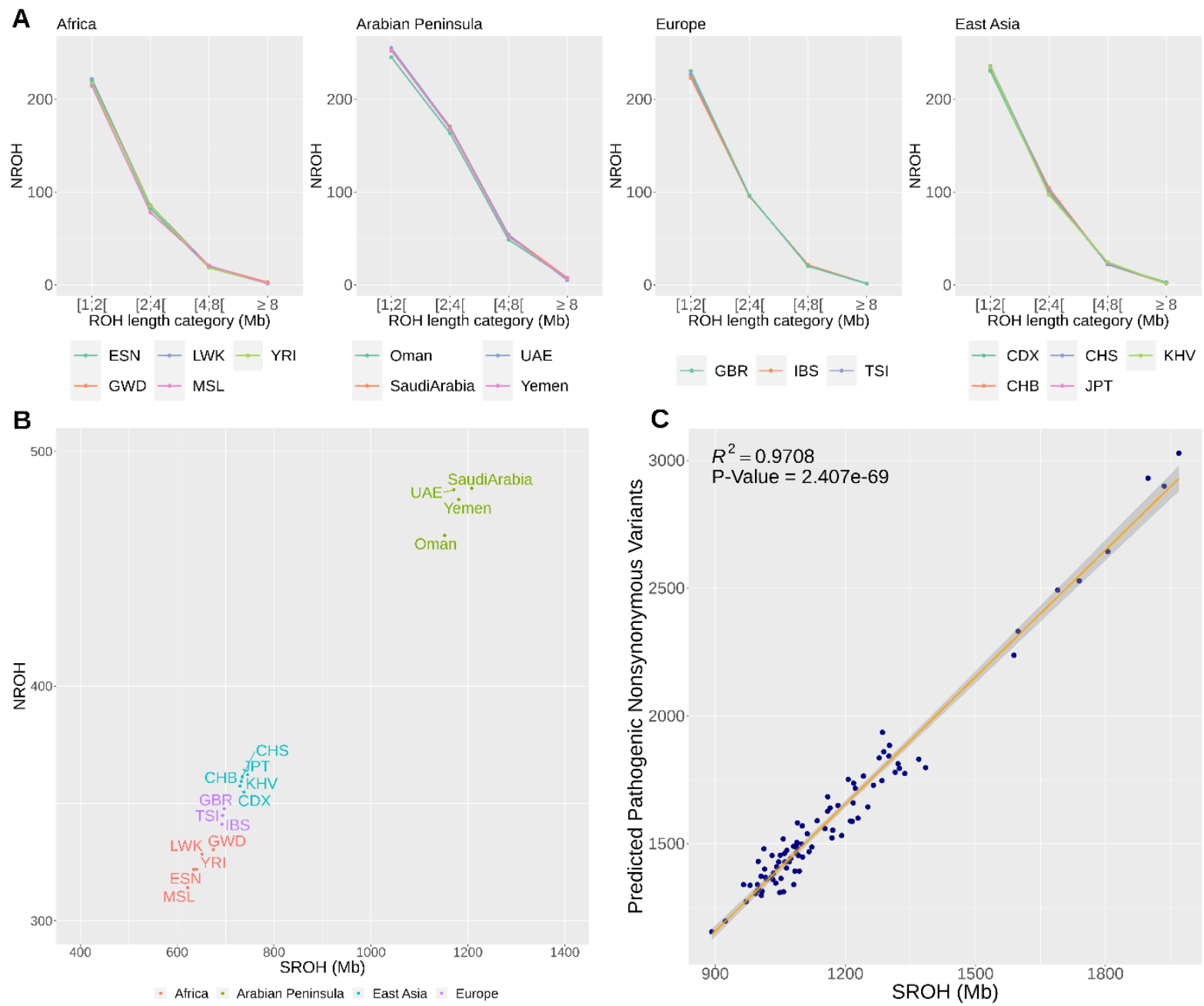

Figure 4: Inbreeding features. A. Mean number of ROHs, NROHs $\geq 1 \mathrm{Mb}$ per population in different length categories, for AP and other globe regions. B. NROHs versus the mean sum $\mathrm{ROH}$ length (SROH) in each region: Africa in red (ESN-Esan in Nigeria, GWD- Mandinka in Gambia, LWK-Luhya in Kenya, MSL-Mende in Sierra Leone, and YRI-Yoruba in Nigeria); AP in green; East Asia in blue (CDX-Dai in China, CHB-Han in China, CHS-Han in South China, JPT-Japanese in Japan, and KHV-Kinh in Vietnam), and Europe in purple (GBR-British in UK, IBS-Iberians in Spain, and TSI-Toscani in Italia). C. Linear regression between SROH and predicted pathogenic non-synonymous variants (for both SIFT and PolyPhen algorithms) in individuals from AP. 


\section{Discussion}

In the present study, we have performed a WES exome capture enriched for UTRs on AP populations, still poorly represented on international WES catalogues. We were able to identify 19,701 new variants $(13.5 \%$ of the total 145,630$)$, that were not catalogued in other public databases, and almost half of these were located in UTR3. We further confirmed that 185 of the UTR variants could be deleterious (inference based on the scaled C-score), being located in genes associated with various types of diseases, especially neurologic and congenital malformation, testifying the importance of screening UTR variants [28,48]. Our results match a previous report [28] focused on the UTR variants from the NHGRI GWAS Catalog [49], which were shown to be mostly associated with immunological, neoplastic and neurological pathologies. As the fine-mapping of UTR regions, especially in terms of their functional impact, is lacking behind the coding regions, there are currently no better pathogenic inferences for these variants than metrics based on conservation. As we improve our knowledge on the functional impact of UTRs, we will refine inferences about their role in diseases. These UTR variants add up to the substantial proportion in the AP WES of 4,673 nonsynonymous variants (15.56\% out of 30,033 total) inferred as pathogenic by SIFT and PolyPhen metrics, and that were distributed in 3,285 genes, $23 \%$ of which have been associated also with neurologic and congenital malformation diseases, as well as other complex disorders.

We have demonstrated that this high burden on pathogenic variants in a relatively low diverse WES cohort (half the level of European and East Asian, and one third of sub-Saharan cohorts) can be explained by the demonstrated considerably high proportion of ROHs due to consanguinity practices. It is known that the strong bottleneck in the OOA dispersal led to a higher burden of pathogenic variants in Europeans and East Asians relative to sub-Saharan Africans [34], but the values for AP are impressive. The low diversity in the AP WES seems contra-intuitive to the identified high admixture in AP populations of sub-Saharan African and South Asian ancestries [13,14,29], but the high consanguinity is strong enough to opose the enrichment with diversity from other population groups. We must reinforce that we were careful in pre-selecting individuals with a main Arabian/NearEast background for WES screening, avoiding Arabian individuals with recent events of admixture. So, values of SROHs would still be more heterogeneous for AP populations if we had included these Arabians with more recent events of admixture. As it is, the main Arabian/NearEast background WES studied here contained several large and homozygous blocks, especially so in Saudi Arabia, where first-cousin marriages are common [16]. Two Saudi Arabian genomes had the highest sum of 
ROHs, as high as $2,000 \mathrm{Mb}$. For contextualisation, the diploid human genome has $6,200 \mathrm{Mb}$, so those Saudi individuals had ROHs across $32 \%$ of their genomes. In mean, the Arabian individuals had ROHs across $19 \%$ of their genomes, against $12 \%$ in East Asians, $11 \%$ in Europeans and 10\% in sub-Saharan Africans. And as we are sequencing coding and regulatory regions of the genome, it is not surprising the high amount of predicted pathogenic variants we found.

Our findings highlight the importance of continuing to catalogue WES in general population cohorts, and in regions of the globe poorly represented in international consortia. The gains definitely pay off the efforts. A contribution of near 20,000 new variants from sequencing around $2 \%$ of the genome in 90 AP individuals is substantial. The pursuit of this cataloguing in populations with high consanguinity is advisable. As we have seen here, the higher the consanguinity the higher the burden of potentially pathogenic variants. These variants are harder to detect in less consanguineous populations due to the effect of genetic drift, which is opposed by consanguinity. In conclusion, we must enrich WES catalogues in ethnic groups and in populations with diverse breeding features, in order to increase the power to robustly identify disease-associated variants in the human species. 


\section{Acknowledgments}

This work was financed by FEDER-Fundo Europeu de Desenvolvimento Regional funds through COMPETE 2020-Operacional Programme for Competitiveness and Internationalisation (POCI), Portugal 2020, by Portuguese funds through FCT-Fundação para a Ciência e a Tecnologia, Ministério da Ciência, Tecnologia e Inovação in the framework of the project "Biomedical anthropological study in Arabian Peninsula based on high throughput genomics" (POCI-01-0145-FEDER-016609). VF has a postdoc grant through FCT (SFRH/BPD/114927/2016). i3S is financed by FEDER-COMPETE 2020, Portugal 2020 and by Portuguese funds through FCT in the framework of the project 'Institute for Research and Innovation in Health Sciences' (POCI-01-0145-FEDER-007274).

\section{Data Availability}

The WES from the individuals analyzed in this study can be accessed from the EGA repository (European Genome-Phenome Archive; accession number: EGA00000000000).

\section{References}

1. DePristo, M.A.; Banks, E.; Poplin, R.; Garimella, K.V.; Maguire, J.R.; Hartl, C.; Philippakis, A.A.; del Angel, G.; Rivas, M.A.; Hanna, M., et al. A framework for variation discovery and genotyping using next-generation DNA sequencing data. Nature genetics 2011, 43, 491-498.

2. Jeroncic, A.; Memari, Y.; Ritchie, G.R.S.; Hendricks, A.E.; Kolb-Kokocinski, A.; Matchan, A.; Vitart, V.; Hayward, C.; Kolcic, I.; Glodzik, D., et al. Whole-exome sequencing in an isolated population from the dalmatian island of vis. European Journal of Human Genetics 2016, 24, 1479-1487.

3. Steyaert, W.; Callens, S.; Coucke, P.; Dermaut, B.; Hemelsoet, D.; Terryn, W.; Poppe, B. Future perspectives of genome-scale sequencing. Acta Clinica Belgica 2018, 73, 7-10.

4. International HapMap Consortium. The international hapmap project. Nature 2003, 426, 789796.

5. Cox, N. Uk biobank shares the promise of big data. Nature 2018, 562, 194-195.

6. $\quad \mathrm{Fu}$, W.; O'Connor, T.D.; Jun, G.; Kang, H.M.; Abecasis, G.; Leal, S.M.; Gabriel, S.; Rieder, M.J.; Altshuler, D.; Shendure, J., et al. Analysis of 6,515 exomes reveals the recent origin of most human protein-coding variants. Nature 2013, 493, 216-220.

7. Lek, M.; Karczewski, K.J.; Minikel, E.V.; Samocha, K.E.; Banks, E.; Fennell, T.; O'Donnell-Luria, A.H.; Ware, J.S.; Hill, A.J.; Cummings, B.B., et al. Analysis of protein-coding genetic variation in 60,706 humans. Nature 2016, 536, 285-291.

8. Karczewski, K.J.; Francioli, L.C.; Tiao, G.; Cummings, B.B.; Alföldi, J.; Wang, Q.; Collins, R.L.; Laricchia, K.M.; Ganna, A.; Birnbaum, D.P., et al. The mutational constraint spectrum quantified from variation in 141,456 humans. Nature 2020, 581, 434-443.

9. Buniello, A.; MacArthur, J.A.L.; Cerezo, M.; Harris, L.W.; Hayhurst, J.; Malangone, C.; McMahon, A.; Morales, J.; Mountjoy, E.; Sollis, E., et al. The nhgri-ebi gwas catalog of 
published genome-wide association studies, targeted arrays and summary statistics 2019. Nucleic Acids Res 2019, 47, D1005-D1012.

10. Pereira, L.; Mutesa, L.; Tindana, P.; Ramsay, M. African genetic diversity and adaptation inform a precision medicine agenda. Nature Reviews Genetics 2021, 22, 284-306.

11. Fernandes, V.; Alshamali, F.; Alves, M.; Costa, M.D.; Pereira, J.B.; Silva, N.M.; Cherni, L.; Harich, N.; Cerny, V.; Soares, P., et al. The arabian cradle: Mitochondrial relicts of the first steps along the southern route out of africa. American journal of human genetics 2012, 90, 347-355.

12. Soares, P.; Alshamali, F.; Pereira, J.B.; Fernandes, V.; Silva, N.M.; Afonso, C.; Costa, M.D.; Musilová, E.; Macaulay, V.; Richards, M.B., et al. The expansion of mtdna haplogroup I3 within and out of africa. Molecular biology and evolution 2012, 29, 915-927.

13. Fernandes, V.; Brucato, N.; Ferreira, J.C.; Pedro, N.; Cavadas, B.; Ricaut, F.-X.; Alshamali, F.; Pereira, L. Genome-wide characterization of arabian peninsula populations: Shedding light on the history of a fundamental bridge between continents. Molecular biology and evolution 2019, 36, 575-586.

14. Fernandes, V.; Triska, P.; Pereira, J.B.; Alshamali, F.; Rito, T.; Machado, A.; Fajkošová, Z.; Cavadas, B.; Černý, V.; Soares, P., et al. Genetic stratigraphy of key demographic events in arabia. PLoS One 2015, 10, e0118625.

15. Almarri, M.A.; Haber, M.; Lootah, R.A.; Hallast, P.; Al Turki, S.; Martin, H.C.; Xue, Y.; TylerSmith, C. The genomic history of the middle east. Cell 2021, 184, 4612-4625.e4614.

16. Almazroua, A.M.; Alsughayer, L.; Ababtain, R.; Al-Shawi, Y.; Hagr, A.A. The association between consanguineous marriage and offspring with congenital hearing loss. Ann Saudi Med 2020, 40, 456-461.

17. Al-Gazali, L.; Hamamy, H.; Al-Arrayad, S. Genetic disorders in the arab world. BMJ (Clinical research ed.) 2006, 333, 831-834.

18. AbdulAzeez, S.; Al Qahtani, N.H.; Almandil, N.B.; Al-Amodi, A.M.; Aldakeel, S.A.; Ghanem, N.Z.; Alkuroud, D.N.; AlTurki, A.; AlQattan, Q.A.; Alghamdi, A., et al. Genetic disorder prenatal diagnosis and pregnancy termination practices among high consanguinity population, saudi arabia. Scientific Reports 2019, 9, 17248.

19. Kirin, M.; McQuillan, R.; Franklin, C.S.; Campbell, H.; McKeigue, P.M.; Wilson, J.F. Genomic runs of homozygosity record population history and consanguinity. PLOS ONE 2010, 5, e13996.

20. Ceballos, F.C.; Joshi, P.K.; Clark, D.W.; Ramsay, M.; Wilson, J.F. Runs of homozygosity: Windows into population history and trait architecture. Nature reviews. Genetics 2018, 19, 220-234.

21. Szpiech, Zachary A.; Xu, J.; Pemberton, Trevor J.; Peng, W.; Zöllner, S.; Rosenberg, Noah A.; Li, Jun Z. Long runs of homozygosity are enriched for deleterious variation. The American Journal of Human Genetics 2013, 93, 90-102.

22. Almaghlouth, I.A.; Hassen, L.M.; Alahmari, H.S.; Bedaiwi, A.; Albarrak, R.; Daghestani, M.; Alqurtas, E.; Alkhalaf, A.; Bedaiwi, M.; Omair, M., et al. National systemic lupus erythematosus prospective cohort in saudi arabia: A study protocol. Medicine 2021, 100.

23. Monies, D.; Abouelhoda, M.; Assoum, M.; Moghrabi, N.; Rafiullah, R.; Almontashiri, N.; Alowain, M.; Alzaidan, H.; Alsayed, M.; Subhani, S., et al. Lessons learned from large-scale, first-tier clinical exome sequencing in a highly consanguineous population. American journal of human genetics 2019, 104, 1182-1201.

24. Project Team, S.G. The saudi human genome program: An oasis in the desert of arab medicine is providing clues to genetic disease. IEEE pulse 2015, 6, 22-26.

25. Wahabi, H.; Fayed, A.; Esmaeil, S.; Mamdouh, H.; Kotb, R. Prevalence and complications of pregestational and gestational diabetes in saudi women: Analysis from riyadh mother and baby cohort study (rahma). BioMed research international 2017, 2017, 6878263. 
26. Rodriguez-Flores, J.L.; Fakhro, K.; Hackett, N.R.; Salit, J.; Fuller, J.; Agosto-Perez, F.; Gharbiah, M.; Malek, J.A.; Zirie, M.; Jayyousi, A., et al. Exome sequencing identifies potential risk variants for mendelian disorders at high prevalence in qatar. Human mutation 2014, 35, 105-116.

27. Scott, E.M.; Halees, A.; Itan, Y.; Spencer, E.G.; He, Y.; Azab, M.A.; Gabriel, S.B.; Belkadi, A.; Boisson, B.; Abel, L., et al. Characterization of greater middle eastern genetic variation for enhanced disease gene discovery. Nature genetics 2016, 48, 1071-1076.

28. Steri, M.; Idda, M.L.; Whalen, M.B.; Orrù, V. Genetic variants in mrna untranslated regions. Wiley interdisciplinary reviews. RNA 2018, 9, e1474.

29. Ferreira, J.C.; Alshamali, F.; Montinaro, F.; Cavadas, B.; Torroni, A.; Pereira, L.; Raveane, A.; Fernandes, $\mathrm{V}$. Projecting ancient ancestry in modern-day arabians and iranians: A key role of the past exposed arabo-persian gulf on human migrations. Genome Biology and Evolution 2021, 13, evab194.

30. Clarke, L.; Fairley, S.; Zheng-Bradley, X.; Streeter, I.; Perry, E.; Lowy, E.; Tassé, A.-M.; Flicek, P. The international genome sample resource (igsr): A worldwide collection of genome variation incorporating the 1000 genomes project data. Nucleic Acids Res 2017, 45, D854-D859.

31. Wang, K.; Li, M.; Hakonarson, H. Annovar: Functional annotation of genetic variants from highthroughput sequencing data. Nucleic Acids Res 2010, 38, e164.

32. Davydov, E.V.; Goode, D.L.; Sirota, M.; Cooper, G.M.; Sidow, A.; Batzoglou, S. Identifying a high fraction of the human genome to be under selective constraint using gerp++. PLOS computational biology 2010, 6, e1001025.

33. Font-Porterias, N.; Caro-Consuegra, R.; Lucas-Sánchez, M.; Lopez, M.; Giménez, A.; CarballoMesa, A.; Bosch, E.; Calafell, F.; Quintana-Murci, L.; Comas, D. The counteracting effects of demography on functional genomic variation: The roma paradigm. Molecular biology and evolution 2021, 38, 2804-2817.

34. Henn, B.M.; Botigué, L.R.; Peischl, S.; Dupanloup, I.; Lipatov, M.; Maples, B.K.; Martin, A.R.; Musharoff, S.; Cann, H.; Snyder, M.P., et al. Distance from sub-saharan africa predicts mutational load in diverse human genomes. Proceedings of the National Academy of Sciences of the United States of America 2016, 113, E440-449.

35. Pollard, K.S.; Hubisz, M.J.; Rosenbloom, K.R.; Siepel, A. Detection of nonneutral substitution rates on mammalian phylogenies. Genome Res 2010, 20, 110-121.

36. Sim, N.-L.; Kumar, P.; Hu, J.; Henikoff, S.; Schneider, G.; Ng, P.C. Sift web server: Predicting effects of amino acid substitutions on proteins. Nucleic Acids Res 2012, 40, W452-W457.

37. Adzhubei, I.A.; Schmidt, S.; Peshkin, L.; Ramensky, V.E.; Gerasimova, A.; Bork, P.; Kondrashov, A.S.; Sunyaev, S.R. A method and server for predicting damaging missense mutations. Nature Methods 2010, 7, 248-249.

38. Kumar, P.; Henikoff, S.; Ng, P.C. Predicting the effects of coding non-synonymous variants on protein function using the sift algorithm. Nature Protocols 2009, 4, 1073-1081.

39. Kircher, M.; Witten, D.M.; Jain, P.; O'Roak, B.J.; Cooper, G.M.; Shendure, J. A general framework for estimating the relative pathogenicity of human genetic variants. Nature genetics 2014, 46, 310-315.

40. Manichaikul, A.; Mychaleckyj, J.C.; Rich, S.S.; Daly, K.; Sale, M.; Chen, W.M. Robust relationship inference in genome-wide association studies. Bioinformatics (Oxford, England) 2010, 26, 2867-2873.

41. Kancheva, D.; Atkinson, D.; De Rijk, P.; Zimon, M.; Chamova, T.; Mitev, V.; Yaramis, A.; Maria Fabrizi, G.; Topaloglu, H.; Tournev, I., et al. Novel mutations in genes causing hereditary spastic paraplegia and charcot-marie-tooth neuropathy identified by an optimized protocol for homozygosity mapping based on whole-exome sequencing. Genetics in medicine : official journal of the American College of Medical Genetics 2016, 18, 600-607.

42. Mamakani, K.; Myrvold, W.; Ruskey, F. Generating all simple convexly-drawable polar symmetric 6-venn diagrams. International Workshop on Combinatorial Algorithms, Victoria. LNCS 2011, 7056, 275-286. 
bioRxiv preprint doi: https://doi org/10.1101/2022.02.22 481461; this version posted February $22,2022$. The copyright holder for this preprint (which was not certified by peer review) is the author/funder, who has granted bioRxiv a license to display the preprint in perpetuity. It is made available under aCC-BY-NC-ND 4.0 International license.

43. Ruskey, F.; Weston, M. A survey of venn diagrams. Electronic Journal of Combinatorics 1997, Dynamic Surveys. Article DS5 (online), Revised 2001 (2005).

44. Wickham, H. Ggplot2: Elegant graphics for data analysis. Springer-Verlag New York: 2016.

45. $\mathrm{R}$ Core Team R: A language and environment for statistical computing., $\mathrm{R}$ Foundation for Statistical Computing: Vienna, Austria, 2018.

46. Landrum, M.J.; Lee, J.M.; Riley, G.R.; Jang, W.; Rubinstein, W.S.; Church, D.M.; Maglott, D.R. Clinvar: Public archive of relationships among sequence variation and human phenotype. Nucleic Acids Res 2014, 42, D980-985.

47. Liu, X.; Wu, C.; Li, C.; Boerwinkle, E. Dbnsfp v3.0: A one-stop database of functional predictions and annotations for human nonsynonymous and splice-site snvs. Human mutation 2016, 37, 235-241.

48. Chatterjee, S.; Pal, J.K. Role of 5'- and 3'-untranslated regions of mrnas in human diseases. Biology of the cell 2009, 101, 251-262.

49. MacArthur, J.; Bowler, E.; Cerezo, M.; Gil, L.; Hall, P.; Hastings, E.; Junkins, H.; McMahon, A.; Milano, A.; Morales, J., et al. The new nhgri-ebi catalog of published genome-wide association studies (gwas catalog). Nucleic Acids Res 2017, 45, D896-D901. 


\section{Figure legends}

Figure 1: The global diversity observed in the 90 AP WES. A. Venn diagram illustrating new and shared variants between AP WES and publicly available databases (as referred in the material and methods section). B. Classes of known variants observed in the AP WES. C. Classes of new variants observed in the AP WES.

Figure 2: Results for the conservation GERP and the integrative scaled C scores. A. Proportion of AP variants from each class of variants in each GERP category. The line represents the total number of variants in each score category (same meaning for B, D and E). B. Proportion of AP variants after normalizing by the total number of variants in each class, observed in that GERP category. C. Proportion of variants from each GERP category in AP and other worldwide regions. GERP values were classified into four groups: neutral $(2<$ GERP), slightly deleterious ( $2<=$ GERP $<4)$, moderate $(4<=$ GERP $<6)$, and extremely deleterious (GERP> 6). D. Proportion of AP variants from each class of variants along the scaled C-scores. E. Proportion of AP variants after normalizing by the total number of variants in each class, along the scaled C-scores. F. Proportion of variants with scaled C-scores below and above 20 (benign and harmful variants, respectively) in AP and other worldwide regions.

Figure 3: Pathogenic predictions. A. SIFT scores versus PolyPhen2 scores. The known variants are represented in light blue dots while new variants are represented as dark blue dots. The yellow dashed line correspond to the thresholds for predicting $0.446<$ Possibly Damaging $<0.908$ for PolyPhen2. The red dashed lines correspond to the thresholds for predicting deleterious variants: $<0.05$ for SIFT and Probably Damaging $>0.908$ for PolyPhen2. B. Diseases associated with 756 of the genes bearing predicted pathogenic (by both SIFT and PolyPhen) non-synonymous variants in AP, according to OMIM (classification of disease based on MalaCards database; Supplementary Table 4). C. Diseases associated with 31 of the genes bearing non-conserved (C-score above 20) UTR3 and UTR5 variants in AP, according to OMIM (classification of disease based on MalaCards database; Supplementary Table 4).

Figure 4: Inbreeding features. A. Mean number of ROHs, $\mathrm{NROHs} \geq 1 \mathrm{Mb}$ per population in different length categories, for AP and other globe regions. B. NROHs versus the mean sum $\mathrm{ROH}$ length (SROH) in each region: Africa in red (ESN-Esan in Nigeria, GWD- Mandinka in Gambia, LWK-Luhya in Kenya, MSL-Mende in Sierra Leone, and YRI-Yoruba in Nigeria); AP in green; East Asia in blue (CDX-Dai in China, CHB-Han in China, CHS-Han in South 
bioRxiv preprint doi: https://doi.org/10.1101/2022.02.22.481461; this version posted February 22,2022 . The copyright holder for this preprint (which was not certified by peer review) is the author/funder, who has granted bioRxiv a license to display the preprint in perpetuity. It is made available under aCC-BY-NC-ND 4.0 International license.

China, JPT-Japanese in Japan, and KHV-Kinh in Vietnam), and Europe in purple (GBR-British in UK, IBS-Iberians in Spain, and TSI-Toscani in Italia). C. Linear regression between SROH and predicted pathogenic non-synonymous variants (for both SIFT and PolyPhen algorithms) in individuals from AP. 